VOL. 38 (1988) [23-30]

\title{
CHARACTERISING COMPLETE BOOLEAN ALGEBRAS IN TERMS OF PURE ESSENTIALNESS
}

\author{
KIRAN R. BHUTANI
}

\begin{abstract}
We discuss purity and pure essentialness of abelian groups in a topos $S h \mathcal{L}$ of sheaves on a locale $\mathcal{L}$ and show that purity is not.a local property. We prove that $A \in A b S h \mathcal{L}$ is divisible if and only if it is pure in every extension, and give an example of a category in which absolutely pure does not imply divisible. We discuss uniform abelian groups and show that each $A U$ uniform in $A b$ does not imply that $A$ is uniform in $A b S h \mathcal{L}$.

Banaschewski showed that the pure subgroups of $Z_{\mathcal{C}}$ are exactly of the type $T_{U} Z_{\mathcal{L}}$ for the different $U \in \mathcal{L}$. We show that $T_{U} Z_{\mathcal{L}}$ is essential in $Z_{\mathcal{L}}$ if and only if $U$ is dense in $\mathcal{L}$, Finally, we characterise as complete boolean algebras the locales for which the only pure and essential subgroup of $Z_{\mathcal{L}}$ is $Z_{\mathcal{L}}$.
\end{abstract}

\section{BACKGROUND}

Definition 1.1: A locale denoted by $\mathcal{L}$ is a complete lattice staisfying the following:

$$
U \wedge \bigvee U_{i}=\bigvee U \wedge U_{i}
$$

for all $U$ and any family $\left\{U_{i}\right\}_{i \in I}$ in $\mathcal{L}$. We denote the minimal element of $\mathcal{L}$ by 0 and the maximal element by $E$. Some examples of locales are a topology of a space, a complete chain, complete boolean algebra or a finite distributive lattice.

Definition 1.2: Recall that for any $0 \neq n \in N$ and $A \in A b S h \mathcal{L}$, one has a map $n_{A}: A \rightarrow A=A \rightarrow A^{n} \rightarrow A$ where $\operatorname{Im} n_{A}$ is denoted by $n A$. Further $A$ is said to be divisible if and only if $A=n A$ for all $0 \neq n \in \mathrm{N}$, that is, for any $a \in A U$ and $0 \neq n \in \mathrm{N}$ there exists a cover $u=\bigvee U_{i}$ such that $a \mid U_{i}=n b_{i}$ for some $b_{i} \in A U_{i}$ for all $i$.

For reference on background material required here, the reader may refer to $[2,4$, $\mathbf{6}, \mathbf{7}, \mathbf{8}, \mathbf{1 0}]$.

\footnotetext{
Received 9 September 1987

Part of this paper is a part of the author's Doctoral dissertation, submitted March 1983 to McMaster University. I am grateful to my supervisor, Professor Bernhard Banaschewski, for his valuable guidance and encouragement throughout the research work. Facilities for the preparation of this paper were provided by the Laboratory of Statistical and Mathematical Methodology at the National Institutes of Health, Bethesda, MD.
}

Copyright Clearance Centre, Inc. Serial-fee code: 0004-9729/88 \$A2.00+0.00. 


\section{PURITY}

Definition 2.1: A monomorphism $h: A \rightarrow B$ in $A b S h \mathcal{L}$ is said to be pure if for all $n \in N$, the diagram

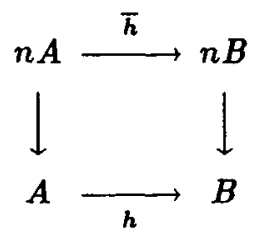

is a pull back diagram. If $A$ is a subgroup of $B$ and $h$ is a natural embedding then $A \subseteq B$ is pure if $n A=A \cap n B$, that is, $(n A) U=A U \cap(n B) U$ for all $U \in \mathcal{L}$.

Counterexample 2.2. The following counterexample shows that purity is not a local property: that is, there is a local $\mathcal{L}$ and $A, B \in A b S h \mathcal{L}$ such that for some cover $E=\bigvee_{i \in I} \ddot{U}_{i}, A\left|U_{i} \subseteq B\right| U_{i}$ is pure in $A b S h \downarrow U i$ for all $i \in I$, but $A \subseteq B$ is not pure in $A b S h \mathcal{C}$. Consider the locale,

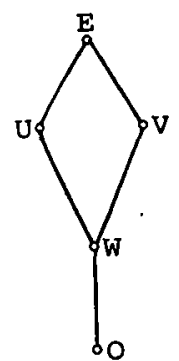

and $A, B \in A b S h \mathcal{L}$ given by,
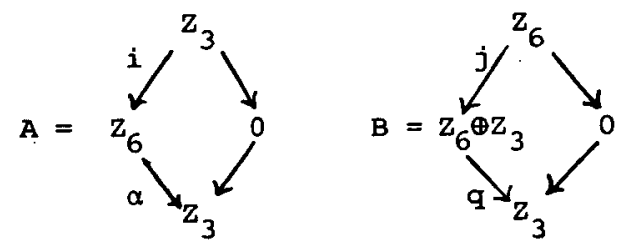

where $\alpha$ is multiplication by 3 and the other maps are obvious maps. Then $A|U \subseteq B| U$ and $A|V \subseteq B| V$ are both pure maps in $A b S h \downarrow U$ and $A b S h\rfloor V$ respectively, but $A \subseteq B$ is not pure in $A b S h \mathcal{L}$, for if this was pure then

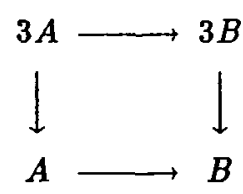


has to be a pull-back which implies that

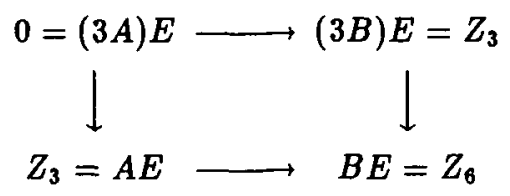

is a pull-back, a contradiction, hence the conclusion.

Proposition 2.3. If $A \in A b S h \mathcal{L}$ is divisible, then for all extensions $B$ of $A$, the monomorphism $h: A \rightarrow B$ is pure.

Proof: If $A$ is divisible then $n A=A$. Hence, it is clear that the diagram

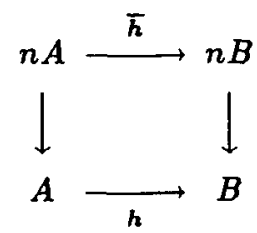

is a pull-back, that is, $h$ is a pure map.

Proposition 2.4. If $h: A \rightarrow B$ is a pure map with $B$ a divisible group, then $A$ is also a divisible group.

Proof: By the given hypothesis, we have a pull back diagram

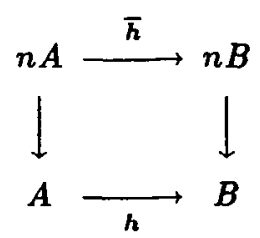

for all $n \in N$. So, there exists a unique $\alpha: A \rightarrow n A$ such that in the diagram,

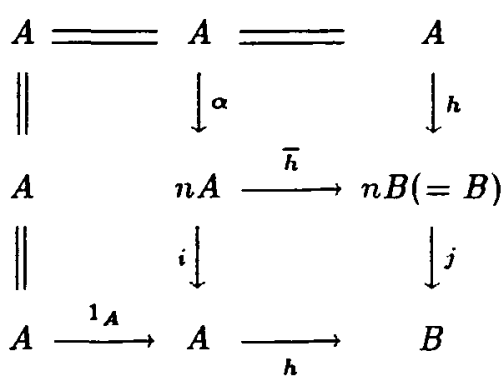

$\bar{h} \alpha=h$ and $i \alpha=1_{A}$. So $i$ is an epimorphism, hence an isomorphism and therefore $A=n A$, that is $A$ is divisible. Thus we obtain the following: 
Corollary 2.5. $A \in A b S h \mathcal{C}$ is divisible if and only if it is pure in every extension.

Proof: $(\Rightarrow)$ This is clear from Proposition 2.3. For the converse, since $A b S h \mathcal{L}$ has enough injectives [10], there exists an injective $B \in A b S h \mathcal{L}$ and a monomorphism $h: A \rightarrow B$, and since injective implies divisible, the result now follows from Proposition 2.4.

Remark 2.6. It is clear that $A \in A b S h \mathcal{L}$ is injective if and only if $A$ is an absolute retract. Since the category $A b S h \mathcal{L}$ has the special property that injective always implies divisible, by the above Corollary we have that injective implies absolute purity. Of course in $A b S h \mathcal{L}, A$ an absolutely pure group does not necessarily imply that $A$ is injective. This is so because for a non-boolean locale $\mathcal{L}$, there are divisible (=absolutely pure) groups which are not injective [1]. Here is an example of an abelian category, where we show that injective does not imply divisible, which also shows that absolutely pure does not imply divisible.

Example 2.7. Consider the category $P$ of elementary abelian $p$-groups. Then $P$ is an abelian category and is the same as the category of vector spaces over the field $Z / p Z$. Therefore each $A \in P$ is an injective group, hence absolutely pure but no nonzero $A$ is divisible, since $0=p A \neq A$.

Proposition 2.8. For $A \rightarrow B$ in $A b S h \mathcal{L}$, if each $A U \rightarrow B U$ is pure in $A b$, then $A \rightarrow B$ is pure in $A b S h \mathcal{L}$.

Proof: This is clear, since the sheaf reflection preserves finite limits and co-limits it preserves pull backs and satisfies the condition $(n A)^{\sim}=n \bar{A}$.

Proposition 2.9. The torsion subgroup of a group is a pure subgroup.

Proof: Let $T$ denote the torsion subgroup of a given group $A \in A b S h \mathcal{L}$. Then $T=\cup_{0 \neq n \in N} \operatorname{Ker} n_{A}$, which is the same as saying that $T U \doteq T(A U)$, where $T(A U)$ is the torsion subgroup of $A U$. Now at each $U \in L$, the diagram

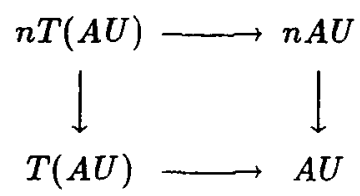

is a pull-back in $A b$, therefore

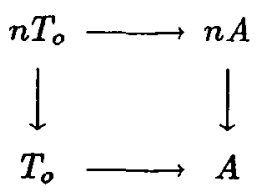


is a pull-back in $A b P S h \mathcal{L}$, where $T_{o}$ is the presheaf $U \rightsquigarrow T(A U)$. By 2.8 it follows that the square

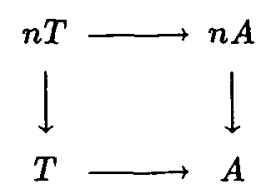

is a pull-back square in $A b S h \mathcal{L}$, hence $T$ is a pure subgroup of $A$.

Proposition 2.10. If $h: A \rightarrow B$ is retractable, then $h$ is pure.

Proof: Let $g: B \rightarrow A$ be such that $g h=1_{A}$. Consider the diagram

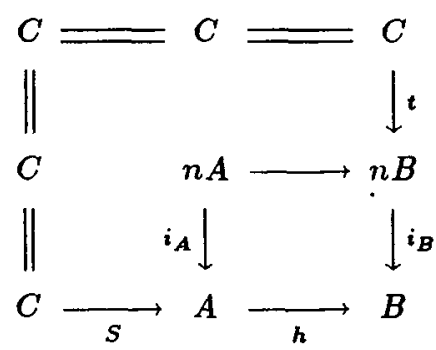

where $h s=i_{B} t$. Now it can be seen as follows that $g h=1_{A}$ implies $\bar{g} \bar{h}=1_{n A}$ (where $\bar{g}: n B \rightarrow n A$ is the unique map such that $i_{A} \bar{g}=g i_{B}$ ). Since $i_{B} \bar{h}=h i_{A}, g i_{B}=i_{A} \bar{g}$, $g i_{B} \bar{h}=g h i_{A}=i_{A}$. Now $i_{A} \bar{g} \bar{h}=g i_{B} \bar{h}=i_{A}=i_{A} 1_{n A}$. Since $i_{A}$ is a monomorphism, $\bar{g} \bar{h}=1_{n A}$. Consider the map $\alpha=\bar{g} t: C \rightarrow n A$. We claim this is the desired map, that is $\alpha$ is unique such that $i_{A} \alpha=s$ and $\bar{h} \alpha=t$. That $\alpha$ is unique is clear, since $1_{A}$ is a monomorphism. Also $i_{A} \alpha=i_{A} \bar{g} t=g i_{B} t=g h s=s$, and $i_{B} \bar{h} \bar{g} t=h i_{A} \bar{g} t=h s=i_{B} t$. Since $i_{B}$ is a monomorphism, it follows $\bar{h} \bar{g} t=\bar{h} \alpha=t$, hence $h: A \hookrightarrow B$ is pure.

Motivated by module theory we say,

Definition 2.11: $A$ in $A b S h \mathcal{L}$ is uniform if every subgroup of $A$ is essential in A.

Proposition 2.12. $A$ is uniform if and only if $E(A)$, the injective hull of $A$, is uniform.

Proof: Let $0 \neq B, C$ be non-zero subgroups of $E(A)$. Since $A$ is an essential subgroup, therefore $A \cap B, A \cap C$ are non-zero subgroups of $A$. But $A$ uniform implies $(A \cap B) \cap(A \cap C) \neq 0$, hence $B \cap C \neq 0$.

Conversely, if $E(A)$ is uniform, then it is clear that every subgroup is uniform.

Proposition 2.13. A uniform implies that $A$ is indecomposable and $A$ an indecomposable injective implies that $A$ is uniform. 
Proof: $(\Rightarrow)$ Let $A=B \oplus C, B \neq 0$, then $A$ uniform and $B \cap C=0$ implies $C=0$.

$(\Leftarrow)$ : Let $0 \neq B \subseteq A$, then $E(B) \subseteq A$ and so $A=E(B) \oplus C$. But $A$ indecomposable implies $E(B)=0$ or $C=0$. Since $0 \neq B$ it follows $C=0$ and so $E(B)=A$. Hence $A$ is an essential extension of each of its subgroups, that is $A$ is uniform.

Counterexample 2.14. If all $A U(U \in \mathcal{C})$ are uniform in $A b$ then $A$ need not be uniform in $A b S h \mathcal{L}$. Consider the locale $\mathcal{L}=3$, and an $A$ in $A b S h 3$ given by

$$
A=\downarrow_{Z}^{Z}
$$

We claim $A$ is not uniform although each $A U=Z$ is uniform in $A b$.

$$
\begin{array}{r}
Z \\
\qquad=\prod_{0}
\end{array}
$$

are non zero subgroups of $A$ but certainly

$$
B \cap C=\downarrow \begin{aligned}
& 0 \\
& \downarrow
\end{aligned}
$$

Proposition 2.15. For any $U \in \mathcal{C}$ the restriction functors $R_{U}: A b S h \mathcal{L} \rightarrow$ AbSh $\downarrow U$ preserve uniform groups.

Proof: Let $A \in A b S h \mathcal{L}$, be uniform. We claim $A \mid U=R_{U} A$ is uniform in $A b S h \downarrow U$. Let $B, C$ be non zero subgroups of $A \mid U$. Then $B W \neq 0$ for $W \subseteq U$ and $C V \neq 0$ for some $V \subseteq U$. This means $E_{U} B$ and $E_{U} C$ are non-zero subgroups of $E_{U}(A \mid U)=T_{U}(A)$. Since $T_{U}(A) \subseteq A[2]$ and $A$ is uniform, $E_{U} B \cap E_{U} C \neq 0$, that is $\left(E_{U} B \cap E_{U} C\right) W$ for some $W \in \mathcal{L}$. By definition of the functor $E_{U}$, [2], there exists some $W_{i} \subseteq W, W_{i} \subseteq U$ such that $(B \cap C) W_{i} \neq 0$, hence $B \cap C \neq 0$, that is $A \mid U$ is uniform in $A b S h \downarrow U$.

Proposition 2.16. Let $C$ be pure in $B$ and $B$ pure in $A$ such that $A$ is a pure exxential extension of $C$. Then $B$ is a pure essential extension of $C$ and $A$ is a pure essential extension of $B$.

Proof: Let $\alpha: B \rightarrow E$ be such that $\alpha i$ is pure where $i: C \rightarrow B$ is the natural embedding. Embed $E$ into its pure injective hull denoted by $\bar{E}$. Since $j: B \rightarrow A$ is 
pure and $\bar{E}$ is pure injective, there exists $\bar{\alpha}: A \rightarrow \bar{E}$ such that $\bar{\alpha} j=k \alpha$.

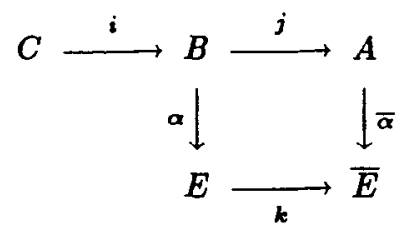

Now $\bar{\alpha} j i=k \alpha i$ and therefore $\bar{\alpha} j i$ is pure. But $j i$ pure essential implies that $\bar{\alpha}$ is a monomorphism and so $\bar{\alpha} j$ is a monomorphism. Since $k \alpha=\bar{\alpha} j, \alpha$ is a mono and hence $i$ is pure essential. Now to see that $A$ is pure essential extension of $B$, we consider $\beta: A \rightarrow D$ such that $\beta j$ is pure. Then $\beta j i$ is pure, but $j i$ pure essential implies $\beta$ is monomorphism, hence $j$ is pure essential.

Remark. In [2], Banaschewski has shown that the pure subgroups of $Z_{\mathcal{L}}$ are exactly of the form $T_{U} Z_{\mathcal{C}}$ for $U \in \mathcal{L}$. The aim is now to characterise those $u \in \mathcal{L}$ for which the pure subgroup $T_{U} Z_{\mathcal{C}}$ is essential.

Proposition 2.17. For any $U \in \mathcal{L}, T_{U} Z_{\mathcal{L}} \subseteq Z_{\mathcal{L}}$ is an essential subgroup if and only if $U$ is dense in $\mathcal{L}$.

Proof: $(\Rightarrow)$ Consider $0 \neq V \in \mathcal{L}$ and $0 \neq a \in Z_{\mathcal{L}} V$. By hypothesis there exist $W \leqslant V$ and $m \in Z$ such that $0 \neq m a \mid W \in\left(T_{U} Z_{\mathcal{L}}\right) W$. By definition of $T_{U} Z_{\mathcal{L}}$, this means there is a cover $W=\bigvee_{i \in I} W_{i}$ such that for some $W_{i} \subseteq U$, $0 \neq(m a \mid W)\left|W_{i}=m a\right| W_{i} \in Z_{\mathcal{L}} W_{i}$. That shows $0 \neq W_{i} \leqslant U \wedge V$, that is $U \wedge V \neq 0$ and therefore $U$ is dense in $\mathcal{L}$.

$(\Leftrightarrow)$ Consider any $0 \neq \phi \in Z_{\mathcal{L}} V$ for some $V \in \mathcal{L}$. Then $V=V_{n \in Z} \phi(n)$, and since $U$ is dense in $\mathcal{L}, 0 \neq U \wedge V=\bigvee_{n \in Z} U \wedge \phi(n)$, therefore $U \wedge \phi(n) \neq 0$ for some $n$. Thus $0 \neq \phi \mid(U \wedge \phi(n)) \in Z_{\mathcal{L}}(U \wedge \phi(n))=\left(T_{U} Z_{\mathcal{L}}\right)(U \wedge \phi(n))$ which shows $T_{U} Z_{\mathcal{L}} \subseteq Z_{\mathcal{L}}$ is essential.

Proposition 2.18. A locale $\mathcal{L}$ is Boolean if and only if the only pure and essential subgroup of $Z_{\mathcal{L}}$ is $Z_{\mathcal{L}}$.

Proof: $(\Rightarrow)$ If $\mathcal{L}$ is Boolean, then $\mathcal{L}$ has no dense elements and so the result follows by 2.17 .

$(\Leftarrow)$ The given conditions imply that $\mathcal{L}$ has no dense elements, hence $\mathcal{L}$ is Boolean.

\section{REFERENCES}

[1] B. Banaschewski, 'When are divisible abelian groups injective?', Quaestiones Math. 4 (1981), 285-307.

[2] B. Bonaschewski, Recovering a space from its abelian sheaves: Seminar talks (McMaster University, 1980). 
[3] K.R. Bhutani, Abelian groups in a topos of sheaves on a locale: Dactoral dissertation (McMaster University, 1983).

[4] K.R. Bhutani, 'Injectivity and injective hulls of abelian groups in a localic topos', Bull. Austral. Math. Soc. 37 (1988), 43-59.

[5] M. Ebrahimi, Algebra in a topos of sheaves: Doctoral dissertation (McMaster University, 1980).

[6] L. Fuchs, Infinite Abelian Groups 1 (Academic Press, New York, 1970).

[7] P. Freyd, Abelian Categories (Harper and Row, New York, 1964).

[8] R. Godement, Theorie des Faisceaux (Hermann, Paris, 1958).

[0] P.T. Johnstone, Stone space: Cambridge Stud. Adv. Math. 3 (Cambridge University Press, 1982).

[10] P.T. Johnstone, Topos theory (Academic Press, New York, 1977).

[11] S. MacLane, Categories for the working mathematician: Graduate Texts in Math. 5 (Springer-Verlag, Berlin, 1971).

Department of Mathematics

The Catholic University of America

Washington D.C. 20064

United States of America 